\title{
CUSTOM DESIGN PERSPECTIVE IN THE PROCESS PARAMETER OPTIMIZATION OF NANO LIPID CARRIERS
}

\author{
ASHWINI M. ${ }^{*}$, PREETHI SUDHEER ${ }^{2}$, BHARANI S. SOGALI ${ }^{3}$
}

1,2Department of pharmaceutics, Krupanidhi College of Pharmacy, Bengaluru, Karnataka, ${ }^{3}$ City Health Care Hospital, Kolar, Karnataka, 5631101 Email: ashwinipreetham2@gmail.com

Received: 28 Aug 2020, Revised and Accepted: 29 Sep 2020

\begin{abstract}
Objective: Nanostructured lipid carrier is an emerging lipid-derived delivery system that is rapidly gaining popularity due to the simplicity of the manufacturing technique. The primary task in formulating nanoparticles is to optimize the parameters that are involved in the process. The
\end{abstract} rationale behind this study is to optimize the process parameters for the preparation of nanostructured lipid carriers.

Methods: The optimization of selected techniques hot homogenization with ultra-sonication and melt dispersion technique, was carried out via statistical analysis software JMP version 13 Pro using custom design approaches. Sonication time, homogenization speed, stirring rate, and cooling temperature were selected as factors for hot homogenization. Stirring speed, stirring time, and dilution volume were the factors deliberated for melt dispersion. The impact of these factors on the responses, particle size, and polydispersity index were studied. The nanoparticles were prepared according to the ten experimental runs generated by the design. Based on the responses, the design space and optimum framework were selected.

Results: The prediction profiler indicated maximum desirability at $81 \%$ and $80 \%$ for hot homogenization and melt dispersion respectively. The actual versus predicted plot of particle size indicated a regression coefficient $\left(\mathrm{R}^{2}\right)$ of 0.98 , and a p-value of 0.0001 for hot homogenization and for melt dispersion the corresponding values were 0.95 and 0.0003 . For the response polydispersity index, these values were 0.92 and 0.0052 for hot homogenization and 0.90 and 0.0024 for melt dispersion.

Conclusion: The endorsing results indicated the authenticity of the model in predicting the significant processing parameters for NLC.

Keywords: Nanostructured lipid carriers, Hot homogenization with ultrasonication, Melt dispersion, Custom design

(C) 2020 The Authors. Published by Innovare Academic Sciences Pvt Ltd. This is an open access article under the CC BY license (http://creativecommons.org/licenses/by/4.0/) DOI: http://dx.doi.org/10.22159/ijap.2020v12i6.39565. Journal homepage: https://innovareacademics.in/journals/index.php/ijap

\section{INTRODUCTION}

Nanostructured lipid carriers (NLCs) are lipid form of nanoparticles which are popular because of its imperfect matrix core of solid lipid and liquid lipid which shield the drug from degradation hence improving its stability $[1,2]$. The lipids used are of generally recognized as safe (GRAS) category which makes it suitable for various routes of administration $[3,4]$.

Several established approaches with rational modification in their methodology have been developed for controlled synthesis of nanoparticles and these approaches can be scaled up $[5,6]$. Optimization of the parameters involved in the process can yield nanoparticles with desired quality attributes [7].

Hence in the present investigation, the selection of optimum processing parameters for the preparation of NLC was the focal point. The two techniques namely hot homogenization technique with ultrasonication (HHU) and melt dispersion technique (MDT) were adopted for preparing NLCs.

Hot homogenization technique with ultrasonication and melt dispersion technique are considered to be the most recommended methods due to a number of advantages such as easy scale-up, product stability, and also eluding the use of organic solvents $[8,9]$.

Custom design helps to build an experimental design that considers the fixed boundary of budget and time. By using this design we can develop a customized framework so that a broad array of challenges can be addressed within a specified scheme. JMP Custom designer permits the consideration of sample size and power calculation by envisaging the alias structure to identify the usefulness through design diagnostic capabilities [10].

\section{MATERIALS AND METHODS}

Glibenclamide was secured as a gift sample from Mankind Pharma India. Stearic acid and oleic acid were furnished by Venus Ethoxyethers Pvt. Ltd India. All additional reagents and chemicals were of analytical status. JMP version 13 Pro was used for the optimization of the parameters.

\section{Experimental design}

Hot homogenization with ultrasonication and melt dispersion are the multifactor approaches that can affect particle size and polydispersity index (PDI) of the product. For hot homogenization with ultrasonication, factors such as sonication time, homogenization speed, stirring rate, and cooling temperature were studied on the responses. For the melt dispersion technique, stirring speed, stirring time, and dilution volume was chosen as factors. Using JMP version 13 Pro, by custom design approach, all factors were selected at two levels low $(-1)$ and high level $(+1)$, and one centre point, was added which generated ten experimental runs.

\section{Preparation of placebo nanostructured lipid carriers}

\section{Hot homogenization technique with ultrasonication}

The procedure was carried out at temperatures above the melting point of the lipids. The solid lipid (stearic acid) was melted at approximately $5-10{ }^{\circ} \mathrm{C}$ above its melting point $\left(69.3^{\circ} \mathrm{C}\right)$. This melted lipid was dispersed in hot liquid lipid (oleic acid) and this hot dispersion was then cascaded to an aqueous phase containing surfactant, tween $20(1.5 \%)$ maintained at the same temperature (above $69.3^{\circ} \mathrm{C}$ ) with constant stirring. This resultant dispersion was then sonicated and further homogenized using polytron homogenizer (Polytron PT 1600 E kinematica homogenizer). This fine emulsion was then poured to the petri dish and cooled to get NLCs $[11,12]$. The ratio of solid lipid to liquid lipid was 70:30. The experimental runs with coded and actual values of factors are outlined in table 1.

\section{Melt dispersion technique}

In the melt dispersion technique, the melted solid lipid (stearic acid) along with hot liquid lipid (oleic acid) was poured into the aqueous phase containing the surfactant (at the same temperature) under stirring to obtain the $0 / \mathrm{W}$ emulsion [13]. This hot dispersion was 
stabilized by stirring for specified periods. The dispersion was then added drop wise into ice-cold water (approximately $10-15^{\circ} \mathrm{C}$ ) under continuous stirring. Once the dispersion attained room temperature, it was filtered and dried $[14,15]$. The ratio of solid lipid and liquid lipid was 70:30. The experimental runs with coded and actual values of factors are depicted in table 2 .

Table 1: Formulation chart (homogenization technique) of placebo NLC as per custom design

\begin{tabular}{|c|c|c|c|c|}
\hline \multirow[t]{2}{*}{ Run } & Stirring rate (rpm) & Homogenization speed (rpm) & Sonication time(min) & Temperature \\
\hline & $\begin{array}{l}-1=500 \mathrm{rpm} \\
0=750 \mathrm{rpm} \\
+1=1000 \mathrm{rpm}\end{array}$ & $\begin{array}{l}-1=15000 \mathrm{rpm} \\
0=17500 \mathrm{rpm} \\
+1=20000 \mathrm{rpm}\end{array}$ & $\begin{array}{l}-1=5 \mathrm{~min}, \\
0=7.5 \mathrm{~min} \\
+1=10 \mathrm{~min}\end{array}$ & $\begin{array}{l}-1=\text { ice bath } \\
+1=\text { Room temperature }\end{array}$ \\
\hline 1 & +1 & -1 & +1 & -1 \\
\hline 2 & -1 & +1 & +1 & 1 \\
\hline 3 & +1 & +1 & -1 & 1 \\
\hline 4 & 0 & 0 & 0 & -1 \\
\hline 5 & +1 & -1 & -1 & 1 \\
\hline 6 & -1 & -1 & -1 & -1 \\
\hline 7 & -1 & +1 & +1 & -1 \\
\hline 8 & +1 & +1 & -1 & -1 \\
\hline 9 & -1 & -1 & -1 & 1 \\
\hline 10 & -1 & -1 & +1 & 1 \\
\hline
\end{tabular}

Table 2: Formulation chart (melt dispersion technique) of placebo NLC as per custom design

\begin{tabular}{|c|c|c|c|}
\hline \multirow[t]{2}{*}{ Run } & Stirring speed(rpm) & Stirring time(min) & Dilution volume(ml) \\
\hline & $\begin{array}{l}-1=600 \mathrm{rpm} \\
0=800 \mathrm{rpm} \\
+1=1000 \mathrm{rpm}\end{array}$ & $\begin{array}{l}(-1=10 \mathrm{~min} \\
0=15 \mathrm{~min} \\
+1=20 \mathrm{~min})\end{array}$ & $\begin{array}{l}(-1=50 \mathrm{ml} \\
0=75 \mathrm{ml} \\
+1=100 \mathrm{ml})\end{array}$ \\
\hline 1 & +1 & +1 & -1 \\
\hline 2 & +1 & +1 & +1 \\
\hline 3 & -1 & +1 & +1 \\
\hline 4 & +1 & +1 & -1 \\
\hline 5 & -1 & -1 & -1 \\
\hline 6 & +1 & -1 & +1 \\
\hline 7 & +1 & -1 & +1 \\
\hline 8 & -1 & -1 & -1 \\
\hline 9 & -1 & +1 & +1 \\
\hline 10 & 0 & 0 & 0 \\
\hline
\end{tabular}

\section{Evaluation of blank formulations}

\section{Particle size analysis and PDI}

SZ-100 HORIBA scientific a particle size, zeta potential, and molecular weight analyser work on the principle of dynamic light scattering (DLS), electrostatic light scattering, and static light scattering technique. The theory of dynamic light scattering was used to determine the mean particle size and particle size distribution of blank NLCs. The samples were diluted with double distilled water and particles were measured in triplicate at a $90{ }^{\circ} \mathrm{C}$ scattering angle and temperature of $25.2^{\circ} \mathrm{C}$ [16].

\section{Analysis of experimental design}

The result of particle size and PDI of all ten experimental trials were substituted into the model and were evaluated further for the authenticity of the model fit. The desirability closer to $100 \%$ was opted for optimizing the process parameters for the responses particle size and polydispersity index [17]. Optimum design space was identified and further drug-loaded formulations were prepared using the optimized factors.

\section{Preparation of drug-loaded formulation}

\section{Hot homogenization technique with ultrasonication}

Glibenclamide was added to stearic acid melt and dispersed in hot oleic acid; then this dispersion was added to aqueous phase containing surfactant, tween 20 with constant stirring. This resultant dispersion was then sonicated and further homogenized using a polytron homogenizer. The fine emulsion was poured into the petri dish and cooled to get the product.

\section{Melt dispersion technique}

Melted solid lipid containing the drug and hot liquid lipid was streamed into the aqueous phase containing tween 20 under stirring to achieve the oil in water emulsion. This hot dispersion was stabilized by stirring and then the dispersion was added drop wise into ice-cold water (approximately $10-15{ }^{\circ} \mathrm{C}$ ) under continuous stirring. Once the dispersion attained room temperature, it was filtered and dried to get the product.

\section{Evaluation of optimized formulations}

\section{Particle size analysis and polydispersity index}

The mean particle size and PDI of optimized drug ladened NLC (GBNLCs) were determined by SZ-100 HORIBA Scientific. The samples were analysed by the same procedure mentioned for the blank formulation [17]

\section{Zeta potential}

Zeta potential is a major characterization required for nanoparticles to approximate its surface charge. It is an essential parameter in the physical stability of NLC. The zeta potential quantification was carried out by SZ-100 HORIBA scientific using the principle of electrostatic light scattering. The samples diluted with double distilled water were placed in the zeta measurement cell. The electrophoretic mobility of the nano dispersion was measured using laser Doppler velocimetry.

\section{Drug entrapment efficacy}

The drug entrapment efficacy (EE) was indirectly obtained by calculating the concentration of free glibenclamide in the supernatant liquid. The optimized GB-NLC (5 mg equivalent) was dispersed in methanol and was centrifuged (Remi motors) at 4000 rpm for $45 \mathrm{~min}$ to segregate the entrapped and unentrapped drug. The supernatant was collected and suitably diluted with methanol and filtered. The filtrate after suitable dilution was measured spectrophotometrically for the drug concentration at $300 \mathrm{~nm}$. The percentage entrapment efficacy of NLC was computed as follows 


$$
\mathrm{EE} \%=\frac{\mathrm{W}_{\mathrm{a}}-\mathrm{W}_{\mathrm{b}}}{\mathrm{W}_{\mathrm{a}}} \times 100
$$

$\mathrm{EE} \%=$ the percentage encapsulation efficiency, $\mathrm{Wa}=$ total drug content of the NLC taken for the study (equivalent to $5 \mathrm{mg}$ ), and $\mathrm{Wb}$ $=$ the quantity of free drug present in the supernatant fluid after centrifugation [18]

\section{Morphological study}

The topical morphology of optimized GB-NLC was studied employing scanning electron microscopy and atomic force microscopy.

\section{Scanning electron microscopy}

The surface morphology of the optimized formulation was studied using the scanning electron microscope (SEM) model EVO MA18 with Oxford EDS (X-act). NLC dispersion was sputtered with gold and spread on a sample holder. The images were obtained at a voltage of $30 \mathrm{kV}$, under argon purging [19].

\section{Atomic force microscopy}

Innova SPM atomic force microscope was used for obtaining images of the samples. Sample tapping mode was used, where the tapping mode maps the topography by lightly tapping the exterior with an oscillating probe tip. The $\mathrm{Z}$ feedback loop was closed to curtail changes in the cantilever's oscillation amplitude with sample surface topography. A minimum of $1 \mu \mathrm{m} \times 1 \mu \mathrm{m}$ area was scanned [20].

\section{Differential scanning calorimetry}

Thermograms were obtained using DSC-60 calorimeter (Shimadzu), the components included a flow controller (FCL 60), a thermal analyzer (TA 60), and operating software (TA 60). A sealed aluminum pan was availed to place the sample, which was flushed with nitrogen $(50 \mathrm{ml} / \mathrm{min})$. The sample was scanned at the rate of 10 ${ }^{\circ} \mathrm{C} /$ min from $20^{\circ} \mathrm{C}$ to $300{ }^{\circ} \mathrm{C}$. An empty aluminum pan was used as a reference [21].

\section{In vitro release study}

In vitro testing of drug release was performed using Franz diffusion cell assembly. In this dialysis technique, the cellophane membrane (Sigma Aldrich) was mounted across the donor and receptor compartment of the assembly, with a diffusion area of $3.14 \mathrm{~cm}^{2}$. The receptor compartment was filled with $50 \mathrm{ml}$ phosphate buffer solution (PBS) pH 7.4 maintained at $37 \pm 0.5{ }^{\circ} \mathrm{C}$ temperature, under the stirring speed of $600 \mathrm{rpm}$. NLC equivalent to $5 \mathrm{mg}$ glibenclamide was suspended in PBS pH 5.5 and placed in the donor chamber. The sample volume of $1 \mathrm{ml}$ was withdrawn at regular time intervals and the sink condition was maintained by replacing fresh buffer into the receptor compartment. The analysis of samples for glibenclamide content was done by UV spectroscopy at $\lambda$ max of $300 \mathrm{~nm}$. A drug dispersion containing $5 \mathrm{mg}$ of drug was studied by the same procedure and comparative data was obtained [22, 23].

\section{RESULTS AND DISCUSSION}

The primary objective of optimization was to fabricate the best formulation under a given set of conditions. Optimization of processing conditions always leads to products with reproducible quality attributes. So NLCs were prepared using two techniques namely HHU and MDT and the optimum processing conditions for the responses such as particle size and polydispersity index were analysed.

\section{Design evaluation}

The responses obtained from the experimental study for the selected factors were substituted into experimental design and the model was evaluated for its suitability of fit under the processing conditions selected. The mean particle sizes for all the ten formulations were within the range of 100 to $200 \mathrm{~nm}$ for both hot homogenization and melt dispersion (table 3 ). The polydispersity index is the indication of particle size distribution in the formulation; a narrow distribution is an essential factor to prove its efficacy. The polydispersity index ranged from 0.110 to 0.5 for both the hot homogenization technique and the melt dispersion technique.

Table 3: Mean particle sizes and polydispersity indices of the formulations

\begin{tabular}{lllll}
\hline Formulation & Mean particle size (nm) & PDI & Mean particle size (nm) & PDI \\
\cline { 2 - 5 } & Hot homogenization & & Melt dispersion & $0.27 \pm 0.33$ \\
F1 & $131 \pm 0.768$ & $0.337 \pm 0.796$ & $155 \pm 0.033$ & $0.129 \pm 0.002$ \\
F2 & $128 \pm 0.673$ & $0.234 \pm 0.234$ & $168 \pm 0.234$ & $0.124 \pm 0.012$ \\
F3 & $112 \pm 0.689$ & $0.28 \pm 0.341$ & $170 \pm 0.087$ & $0.27 \pm 0.012$ \\
F4 & $126 \pm 0.754$ & $0.186 \pm 0.367$ & $157 \pm 0.122$ & $0.247 \pm 0.43$ \\
F5 & $125 \pm 0.987$ & $0.43 \pm 0.145$ & $175 \pm 0.108$ & $0.11 \pm 0.017$ \\
F6 & $124 \pm 0.901$ & $0.129 \pm 0.456$ & $178 \pm 0.345$ & $0.12 \pm 0.0321$ \\
F7 & $133 \pm 0.876$ & $0.178 \pm 0.364$ & $180 \pm 0.098$ & $0.249 \pm 0.154$ \\
F8 & $119 \pm 0.912$ & $0.123 \pm 0.312$ & $174 \pm 0.087$ & $0.126 \pm 0.037$ \\
F9 & $121 \pm 0.980$ & $0.129 \pm 0.123$ & $169 \pm 0.008$ & $0.264 \pm 0.0456$ \\
F10 & $129 \pm 0.870$ & $0.27 \pm 0.786$ & $175 \pm 0.421$ & \\
\hline
\end{tabular}

Notes: Data given in mean $\pm \mathrm{SD}, \mathrm{n}=3$

The Analysis of variance (ANOVA) (table 4) gives information about effect of each factor, its interactions and the significant levels of these factors on the responses. The model fit is first evaluated using adjusted $R^{2}$ value and $p$ value [24]. $p$ value of $5 \%$ or less by ANOVA and values close to one for $R^{2}$ indicates the level of significance and correlation between predicted and actual responses.

Table 4: ANOVA results for particle size and polydispersity index

\begin{tabular}{lll}
\hline Term & Prob $>|\mathbf{t}|$ \\
\cline { 2 - 3 } & Particle size \\
\hline Hot homogenization technique with ultrasonication & $<0.0001^{*}$ & PDI \\
Sonication time(5,10) & $0.0343^{*}$ & $0.0043^{*}$ \\
H speed(15000,20000) & $0.0014^{*}$ \\
Stirring rate $(500,1000)$ & $0.0006^{*}$ & $0.0038^{*}$ \\
Temperature & & $0.0278^{*}$ \\
Melt dispersion technique & $0.0428^{*}$ \\
Stirring speed(600,1000) & $0.0001^{*}$ \\
Stirring time(10,20) & $0.0011^{*}$ \\
Dilution volume(50,100) & \\
\hline
\end{tabular}


For the hot homogenization technique, all the parameters had a significant influence on the response particle size; sonication time and temperature showed the highest significance, followed by stirring rate and homogenization speed. The polydispersity index was highly affected by the stirring rate and sonication time. Temperature also had a significant influence but the impact of homogenization speed on PDI was not noteworthy as per the observations. Shear intensity is an important factor as it causes the deformation of particles and may also accelerate the formation of foam or aggregation, hence optimum shear intensity is vital for attaining the required particle size with satisfactory polydispersity index [25].
In melt dispersion, the particle size was significantly influenced by stirring time, dilution volume, and stirring speed. The polydispersity index was significantly influenced only by the dilution factor $(\leq 0.05)$. Stirring time and stirring speed had very less impact on PDI.

The actual verses prediction plot signifies the effect of the model and compares it against the null model. If the points are close to the fitted line, with constricted confidence bands the model is considered to be a good fit.

The actual verses prediction plot for the responses particle size and PDI of homogenization technique and melt dispersion technique is given in fig. $1 \mathrm{a}, 1 \mathrm{~b}, 2 \mathrm{a}$ and $2 \mathrm{~b}$.

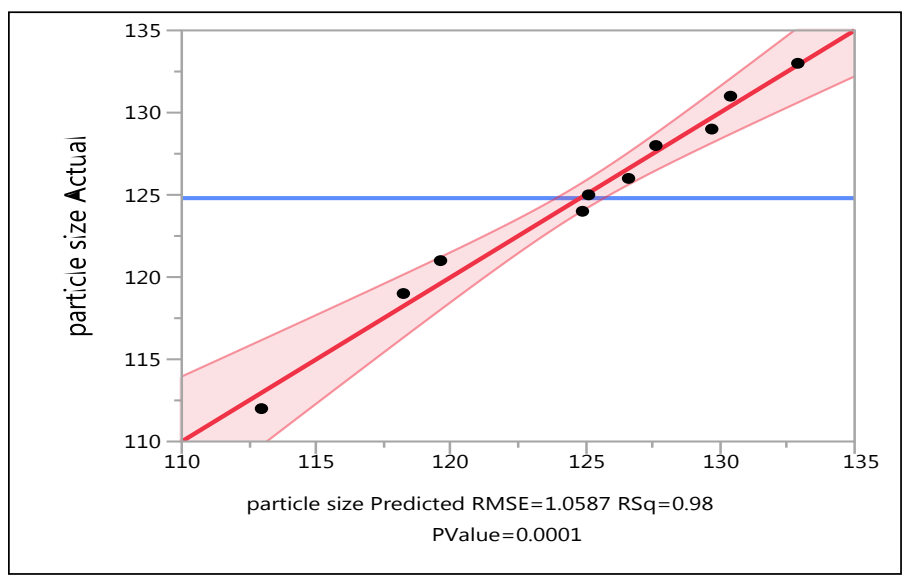

Fig. 1a: Actual vs predicted plots of the particle size for hot homogenization technique

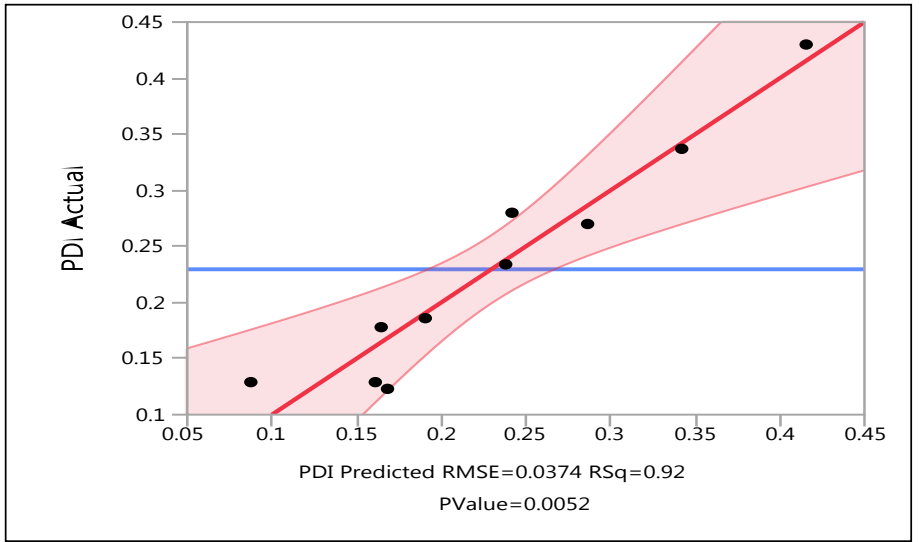

Fig. 1b: Actual Vs predicted plots of PDI for hot homogenization technique

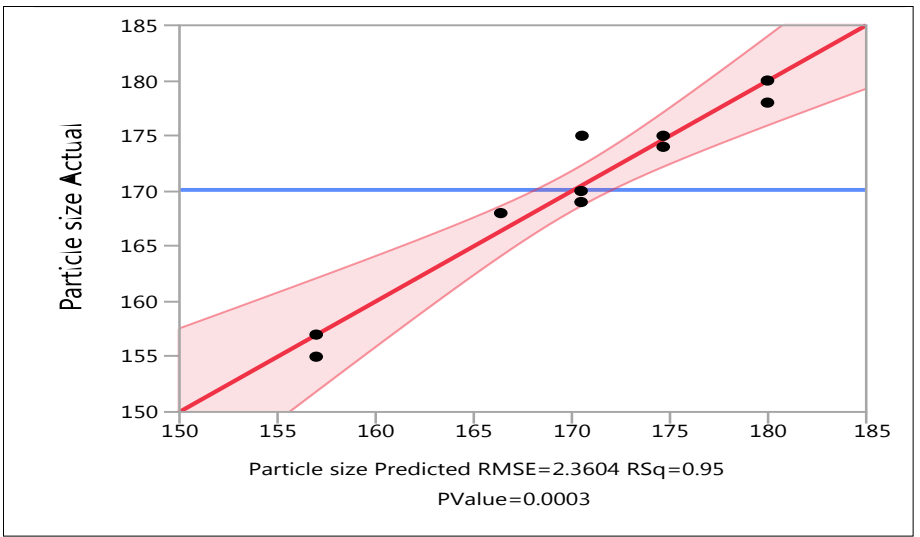

Fig. 2a: Actual Vs predicted plots of the particle size for melt dispersion technique 


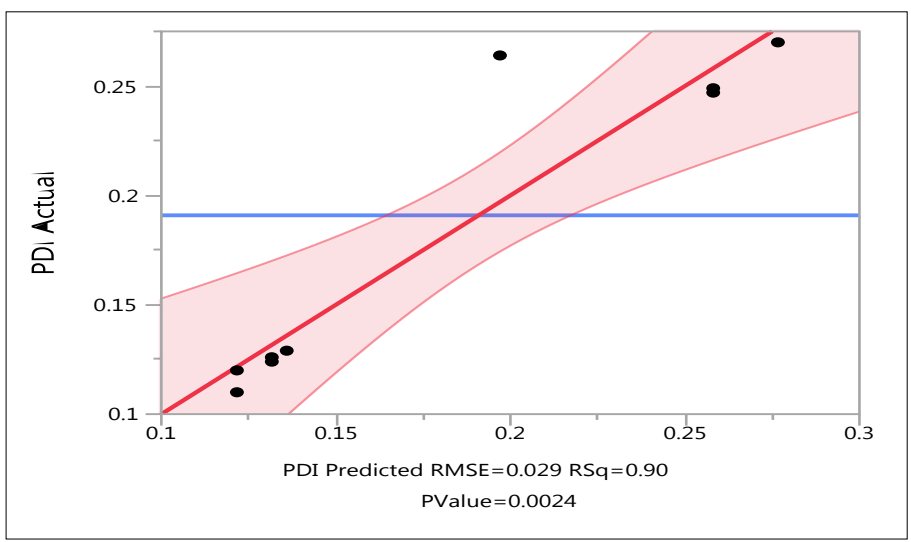

Fig. 2a: Actual Vs predicted plots of PDI for melt dispersion technique

According to the predictive models of the homogenization technique the values got for particle size $\left(p=0.0001, R^{2}=0.98\right)$ and PDI ( $p=0.0052, R^{2}=0.92$ ) confirms the validity of the model, data points are found to be almost linear for particle size and PDI.

As per the predictive models of melt dispersion technique, the values attained for particle size $\left(p=0.0003, R^{2}=0.95\right)$ and PDI $\left(p=0.0024, R^{2}=90\right)$ indicated that observed values were in close conformity with the predicted estimates. The confidence bands of PDI were slightly broader compared to that of particle size which can be attributed to a few outlier points in the PDI graph.

Desirability methodology is an influential optimization tool. The values vary from 0 to 1 . A scale-free value is obtained from response transformation [26]. This approach specifies the quality of a process or a product that depends on the characteristics of factors chosen. It gives the operating conditions that provide the most desirable response. The value of desirability close to one is considered to be ideal and close to zero is a non-ideal condition [27].

The maximum desirability for hot homogenization was observed at (0.81) i.e. $81 \%$ (fig. 3) at a homogenization speed of $17500 \mathrm{rpm}$, sonication time of $7.5 \mathrm{~min}$, stirring rate of $750 \mathrm{rpm}$ and ice bath temperature and the predicted particle size was $126 \mathrm{~nm}$ and the polydispersity index was 0.190

For the melt dispersion technique, the maximum desirability was $(0.804)$ or $80 \%$ (fig. 4 ) observed at a stirring speed of 800 rpm, stirring time of $15 \mathrm{~min}$, and dilution volume of water $75 \mathrm{ml}$ which predicted a particle size of $170 \mathrm{~nm}$ and PDI of 0.197.

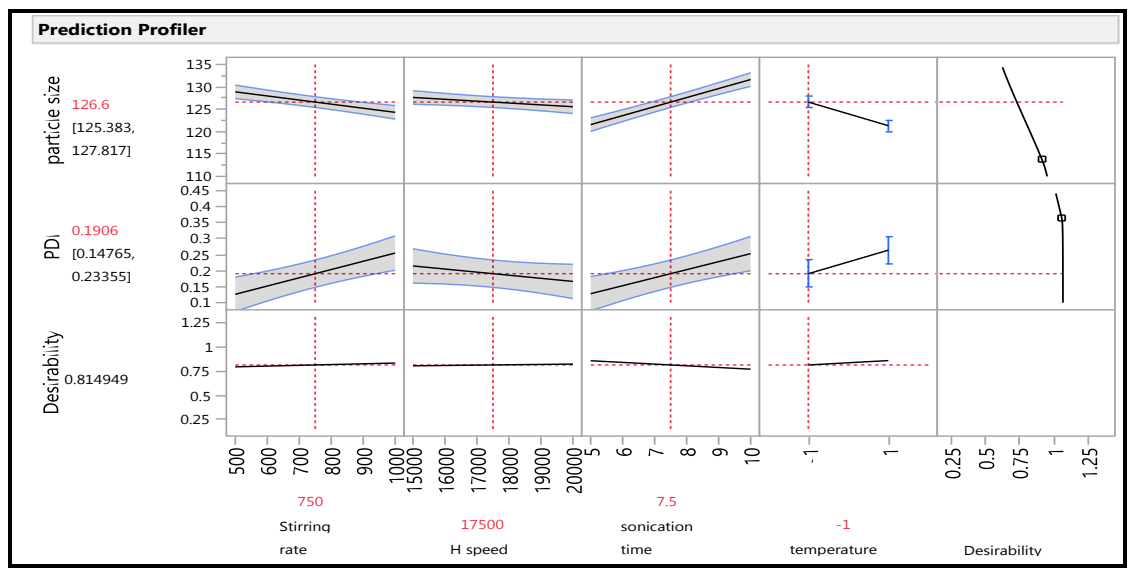

Fig. 3: Prediction profiler of optimized process parameters for hot homogenization technique

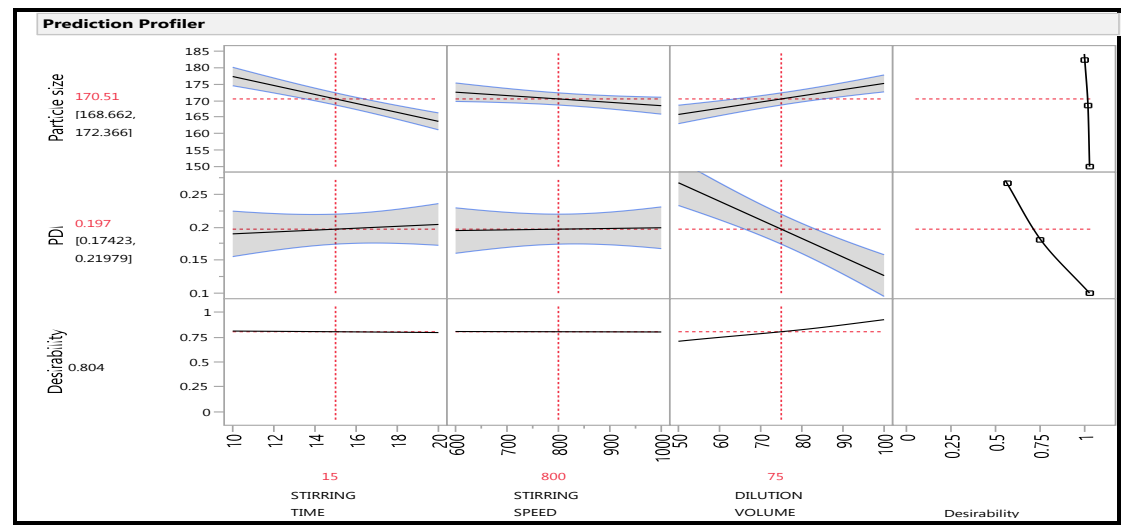

Fig. 4: Prediction profilers of optimized process parameters for melt dispersion technique 


\section{Prediction expression for particle size}

The prediction expression generated for particle size of NLC developed by HHT (equation 1) and MDT (equation 2) shown below

$123.9634615=-2.28846153846154 *($ Stirring rate-750)/250 $)+(-$ $1.03846153846154 *$ (H speed-17500/2500)+5.03846153846154* (sonication time-7.5)/2.5)+(-2.63653846153846* temperature)-Eq. (1).

According to the expression stirring rate, homogenization speed and temperature had a negative impact on particle size. An increase in these parameters causes a decrease in the particle size. Whereas sonication time presented a positive upshot.

$170.514018691589=+-6.79672897196262 *$ (stirring time15)/5)+-2.04672897196262* (stirring speed-800)/200)+ $4.70327102803738 *$ (dilution volume-75)/25)]-Eq. (2).

Among the significant factors stirring time, stirring speed showed a positive effect and vice versa for dilution volume

\section{Prediction expression for PDI}

Prediction formula expressed for PDI of NLC developed by HHT (equation 3) and MDT (equation 4) is as follows

$0.227367307692308=+0.0645576923076923 *$ (Stirring rate-750) $/ 250)+(-0.0241923076923077 *$ (H $\quad$ speed-17500)/2500)+ $0.0626923076923077^{*}$ (sonication

$0.0367673076923077 *$ temperature----Eq. (3).
Stirring rate, sonication time and temperature are factors significantly influencing PDI according to the $p$-value and all these values exhibited a positive impact.

$0.19700934579=+0.0071355140186916 *$ (stirring time15)/5)+0.00213551401869159* (stirring speed-800)/200)+$0.0703644859813084^{*}$ (dilution volume-75)/25)]-Eq. (4).

The expression indicated a negative impact of the significant factor dilution volume on PDI

Characterization of the NLC formulated at optimized process parameters

The optimized drug (glibenclamide $20 \mathrm{mg}$ ) loaded NLCs prepared by homogenization method (GB-NLC1) and melt dispersion technique (GB-NLC2) were assessed for particle size, PDI, zeta potential, drug entrapment, SEM, AFM, DSC, and in vitro drug release

\section{Particle size and polydispersity index}

Particle size determination is one of the important evaluation parameters for NLCS; as it pays for greater area and aids for better drug absorption. Also particle size and PDI indicates the steadiness of the product which is illustrated in fig. 5 and fig. 6 [28]. The particle size of (GB-NLC1) and (GB-NLC2) was found to be $128 \pm 0.254 \mathrm{~nm}$ and $169 \pm 0.435 \mathrm{~nm}$ which was within the design space. The results of PDI (GB-NLC1: 0.188 \pm 0.098 ; GB-NLC2: $0.2 \pm 0.018$ ) was closer to that predicted and indicates a narrow size distribution of the nanoparticles.

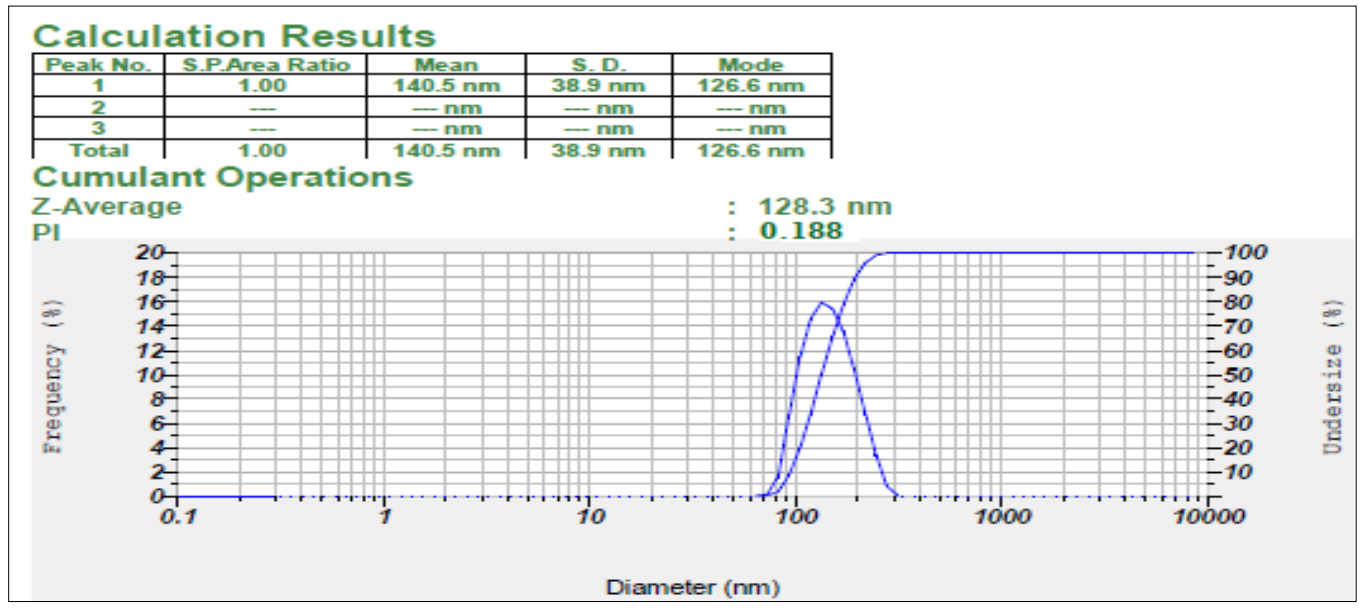

Fig. 5: Particle size and PDI of optimized formulation GB-NLC1 (mean \pm SD, $n=3$ )

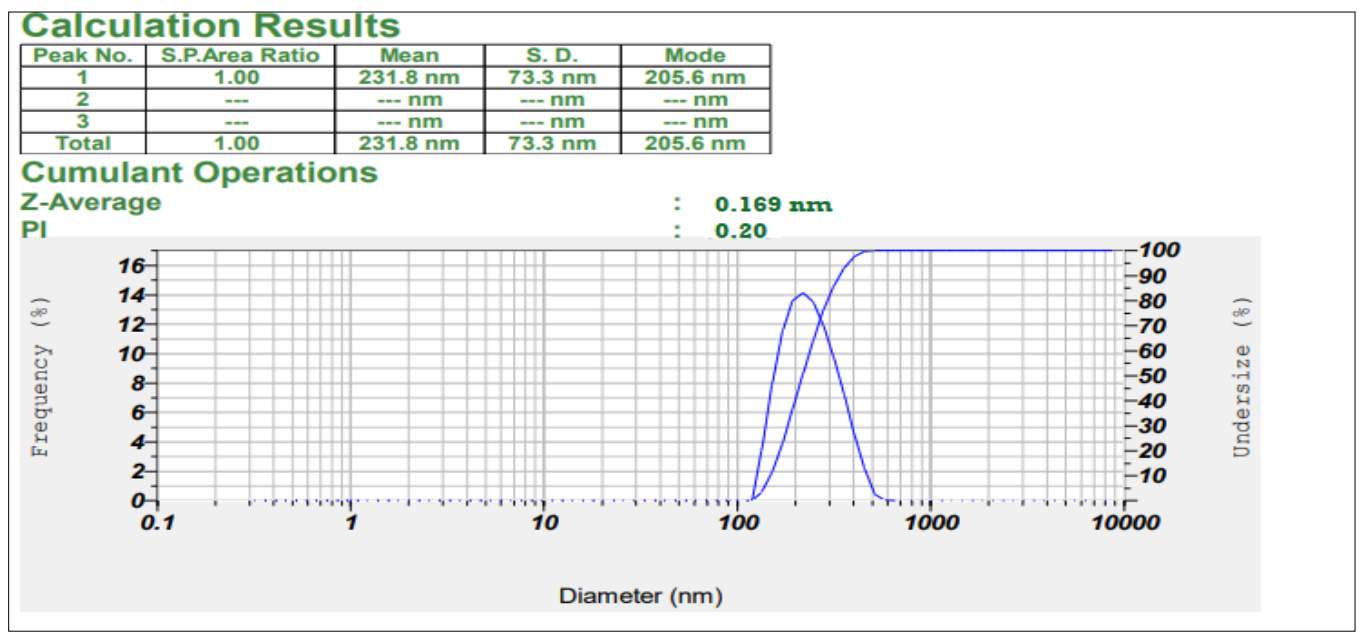

Fig. 6: Particle size and PDI of optimized formulation GB-NLC2 (mean $\pm S D, n=3$ ) 


\section{Zeta potential}

The surface charge on the nano carriers is determined by computing the zeta potential (ZP). ZP grades electrostatic repulsion amongst similarly charged constituent in the dispersion. For nano particles higher zeta potential gives stability by avoiding accumulation. Particulates begin to aggregate or flocculate when the zeta values are lowered [29]. The zeta potential of $\geq 30 \mathrm{mV}$ designates the stability of any dispersed systems. The zeta potential of GB-NLC1 and GB-NLC2 was- $38.0 \mathrm{mV}$ and-49 mV respectively and depicted in fig. 7 and fig. 8 . This indicates an effective electrostatic repulsion between particles which resulted in the separation of the particle and thus preventing agglomeration.

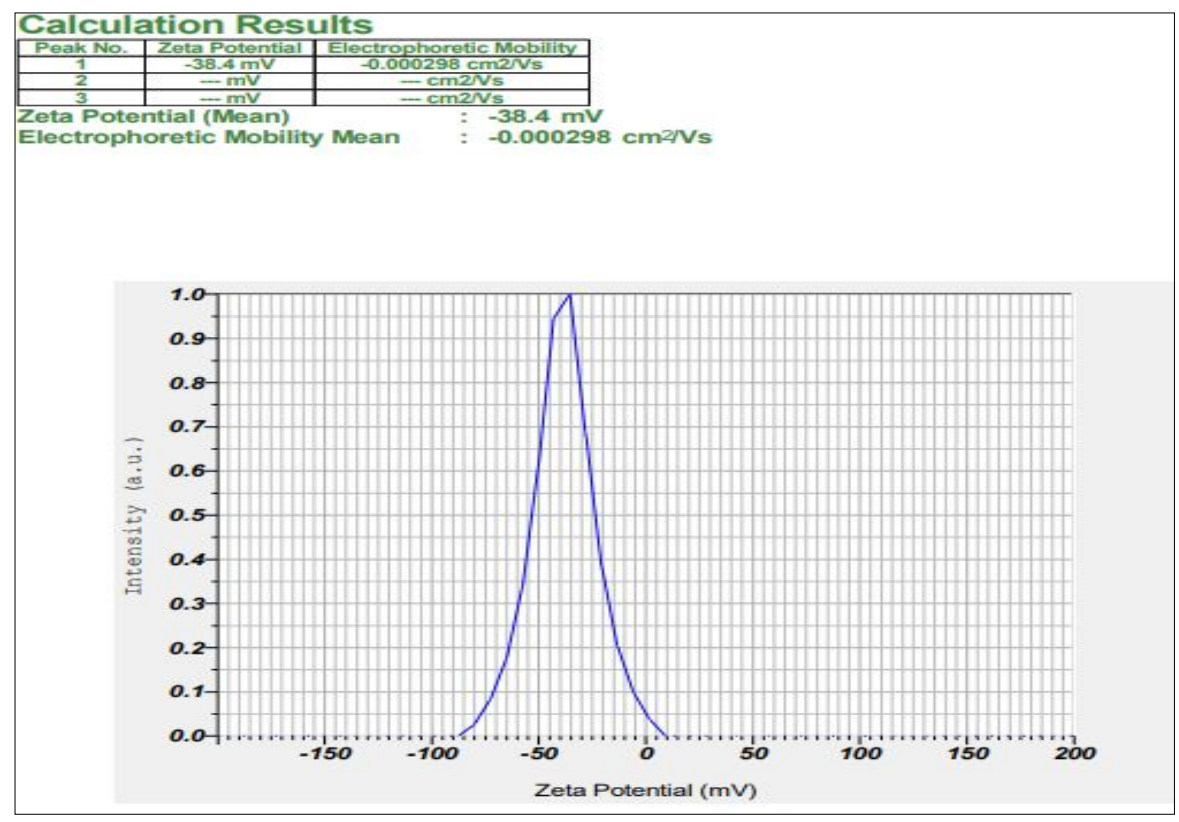

Fig. 7: Zeta potential of optimized formulation GB-NLC1 (mean $\pm S D, n=3$ )

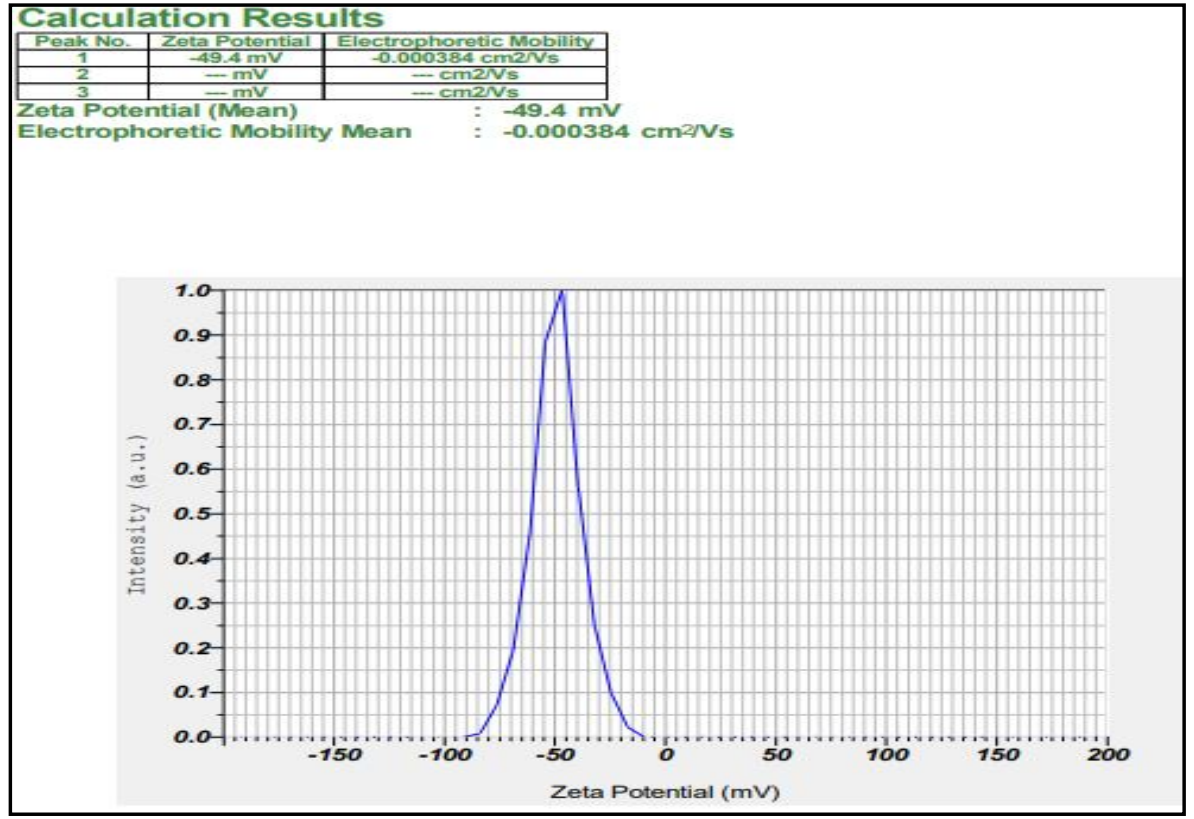

Fig. 8: Zeta potential of optimized formulation GB-NLC2 (mean $\pm S D, n=3$ )

\section{Drug entrapment}

The drug entrapment was observed to be $94.1 \% \pm 0.678$ for GB-NLC1 and $92.6 \% \pm 0.456$ for GB-NLC2 this may be endorsed to the distorted lipid core of optimized NLC due to blending solid lipid with liquid lipid. This amalgamation of solid and liquid lipid leads to increased loading of drug and minimizes drug expulsion and loss during storage.

\section{Morphological study}

Drug loaded formulations appeared distinct as demonstrated in SEM (fig. 9a and fig. 9b) and AFM images (fig. 10a and fig. 10b) and the size was in the nano meter range which complies with the particle size data derived from dynamic light scattering (DLS) system. Few agglomeration seen may be accounted for the sticky nature of the lipids in the formulation [30]. 


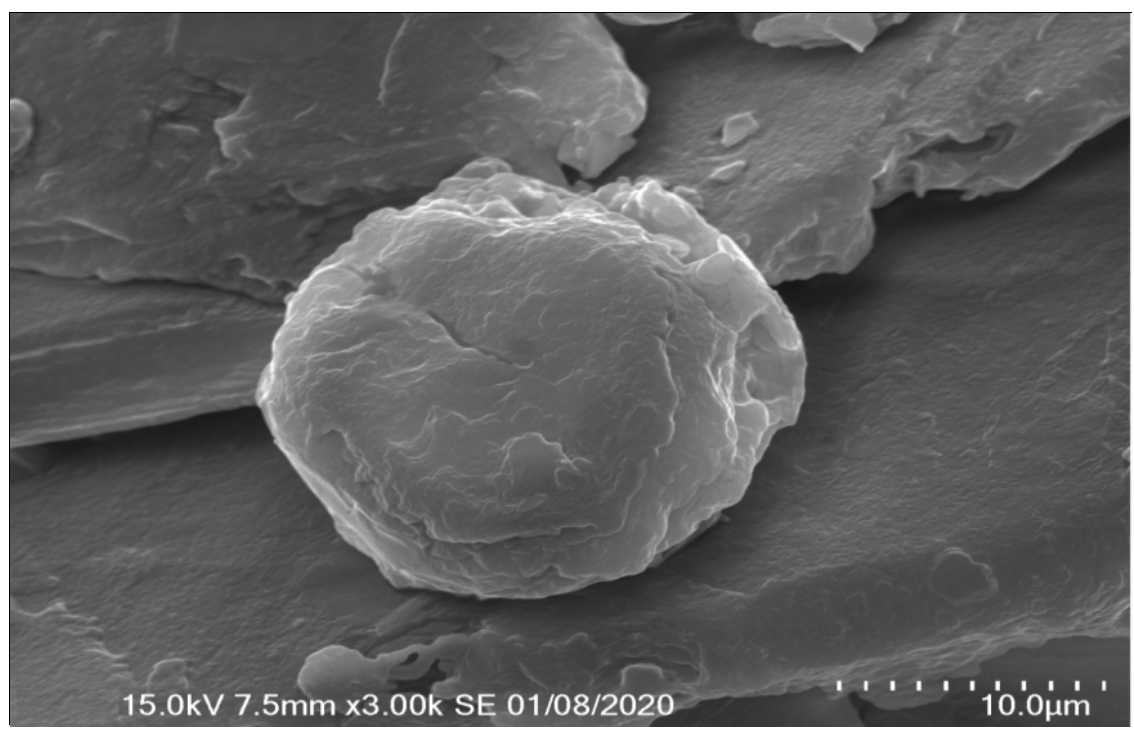

Fig. 9a: SEM image of optimized formulation GB-NLC1

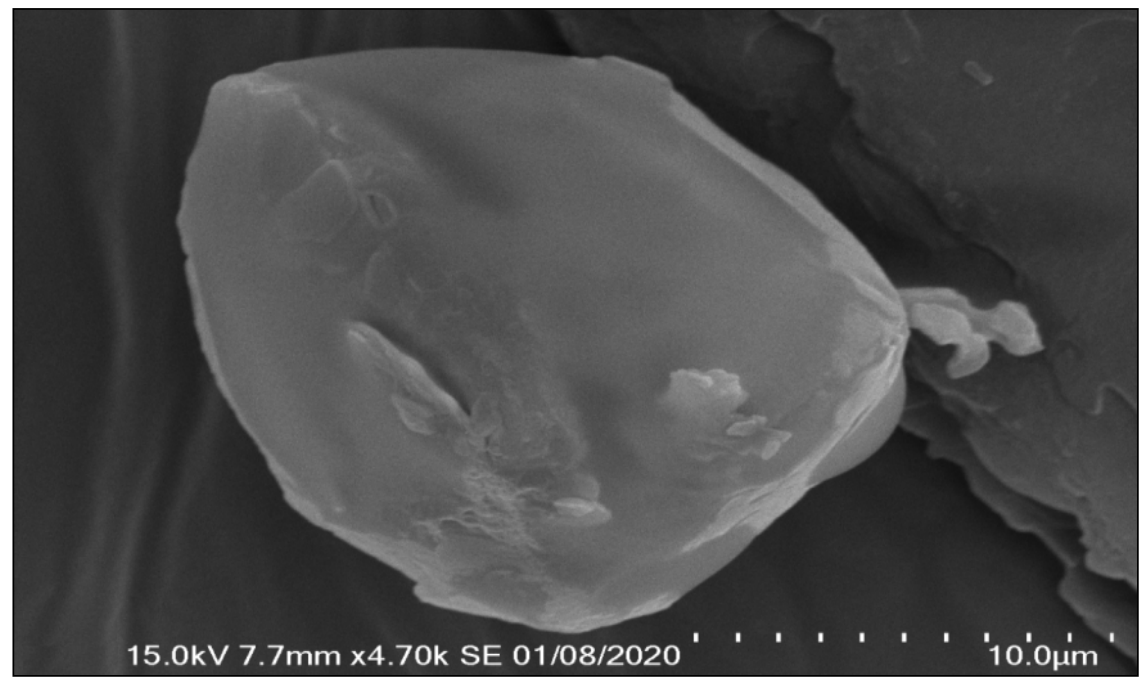

Fig. 9b: SEM image of optimized formulation GB-NLC2

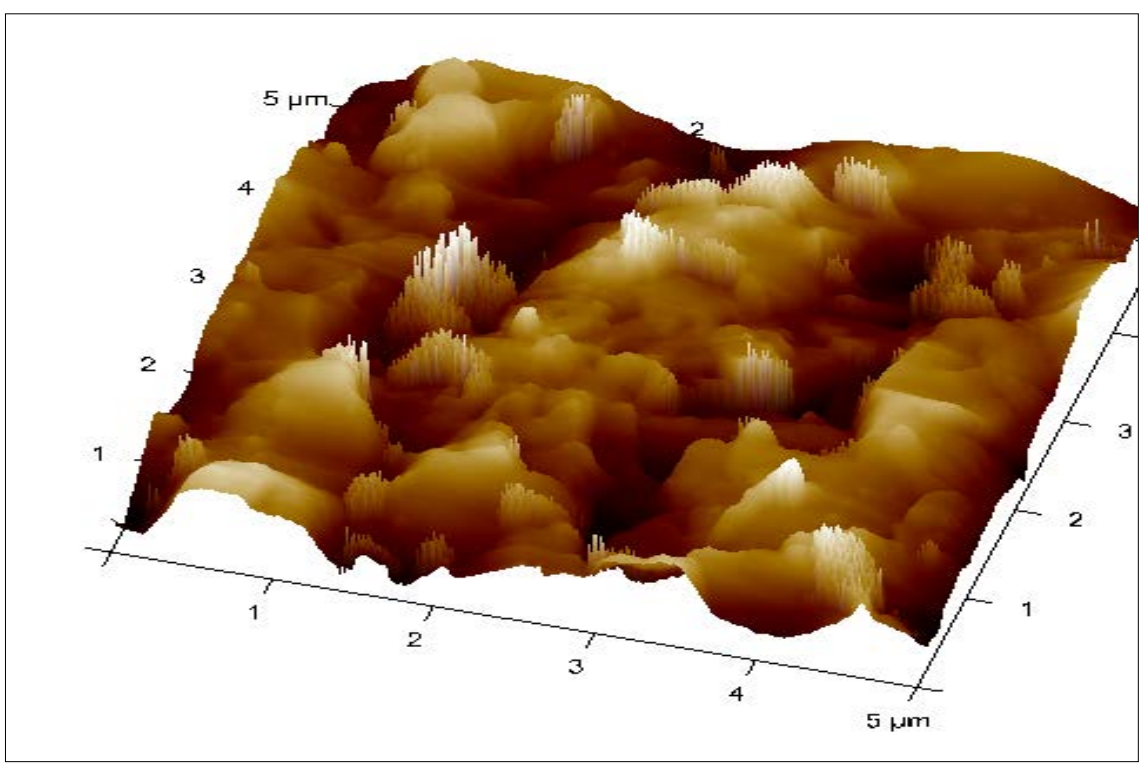

Fig. 10a: AFM image of optimized formulation GB-NLC1 


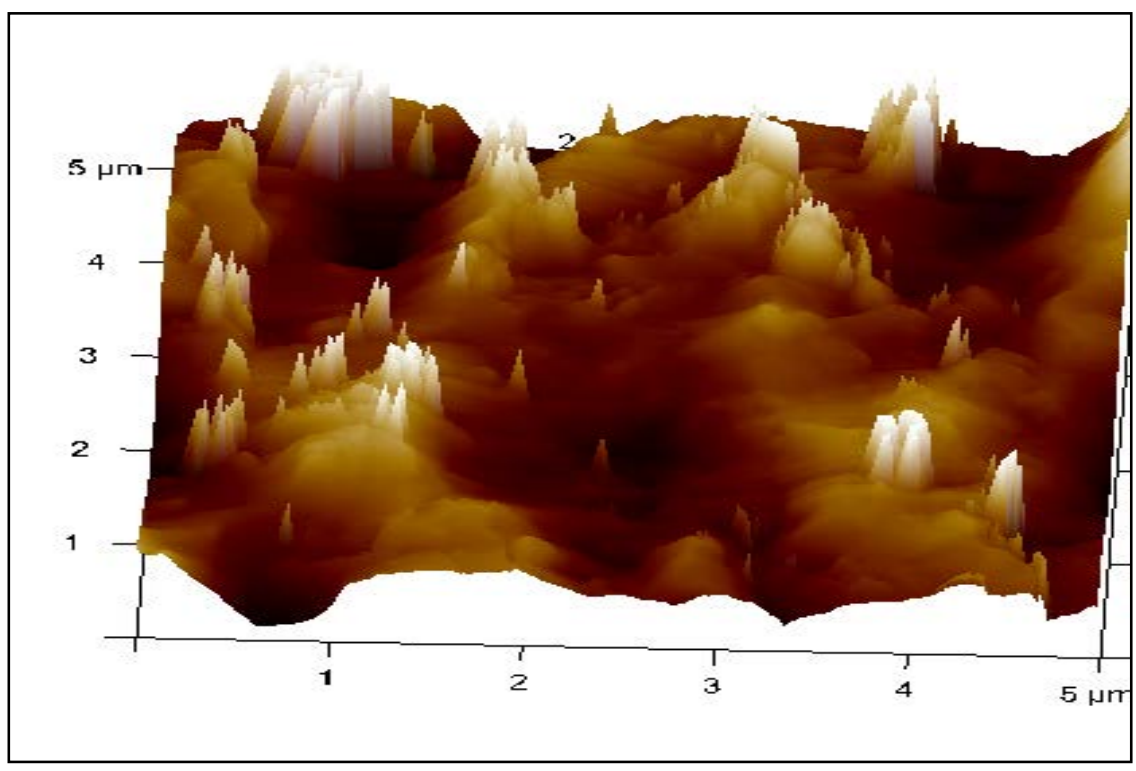

Fig. 10b: AFM image of optimized formulation GB-NLC2

\section{Differential scanning calorimetry}

The DSC study was carried out for the optimized GB-NLC1 and GBNLC2, glibenclamide, physical mixture (drug, solid lipid, liquid lipid, and surfactant), and the thermograms are presented in fig. 11. DSC thermograms of GB showed a melting peak at $171.35^{\circ} \mathrm{C}$ which is an indication of the high crystalline structure of GB. The physical mixture showed a peak at $169^{\circ} \mathrm{C}$ nearer to the melting peak of GB. The peak of optimized GB-NLC1 was seen at $42.5^{\circ} \mathrm{C}$ and that of GBNLC2 was at $38.42{ }^{\circ} \mathrm{C}$, which is a clear indication that GB was molecularly distributed in the lipid array of the formulated optimized NLC. The addition of the drug to the melted lipid and the cooling temperature may have contributed to this less ordered matrix of the formulation [31].

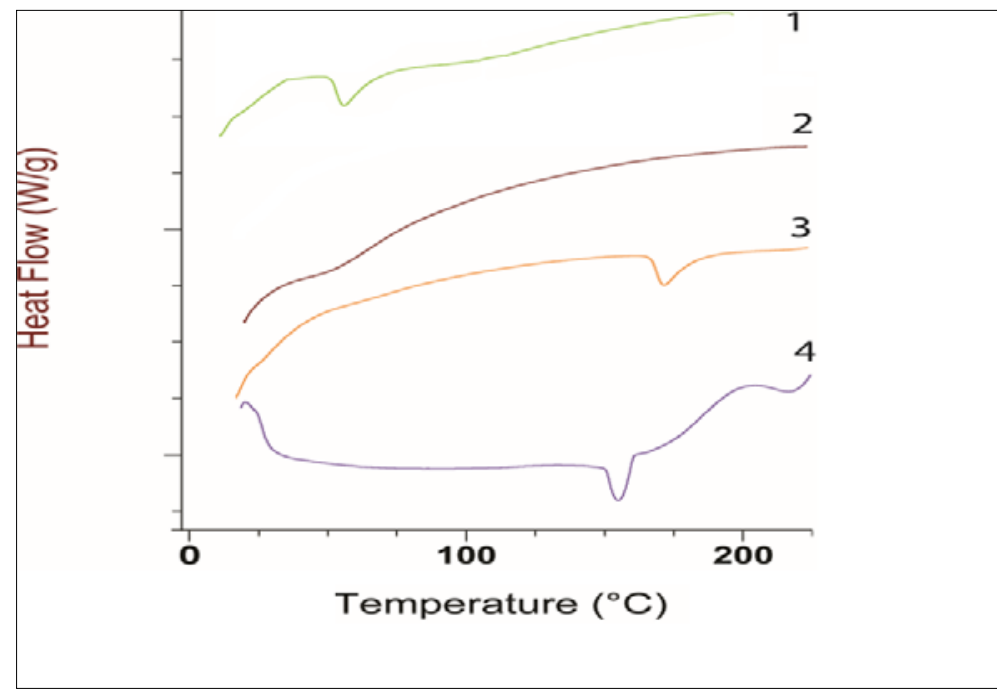

Fig. 11: DSC thermograms of (1) optimized GB-NLC1, (2) optimized GB-NLC2 (3) glibenclamide, (4) physical mixture

\section{In vitro release study}

The in vitro release characteristics of glibenclamide from optimized GB-NLC1 and GB-NLC2 against drug dispersion (GB dispersion was used as a reference for comparison) were observed for a period of 8 $\mathrm{h}$ as illustrated in the fig. 12. It is seen that GB released from optimized NLC showed an initial quick-release followed by a slowrelease. The initial rapid release of the drug may be due to the release of GB from the NLC facet, while at a later juncture GB was regularly released from the nucleus of NLCs. The facet drug concentration may be attributed to the cooling of particles from higher to lower temperatures, which might have promoted the accumulation of drug in the outer surface of the formulation leading to the preliminary burst release.

GB dispersion showed a faster release in comparison to the GBNLC1, and GB-NLC2. However, the release from GB-NLC1 was prolonged up to $93 \% \pm 0.173$ and from GB-NLC2 it was prolonged to $89.5 \% \pm 0.707$ up to $8 \mathrm{~h}$ when compared to the GB dispersion which released $92.5 \% \pm 0.981$ in $4 \mathrm{~h}$. This improvement can be attributed to the existence of solid and liquid lipid in the NLC system which ameliorated the release prospective. 


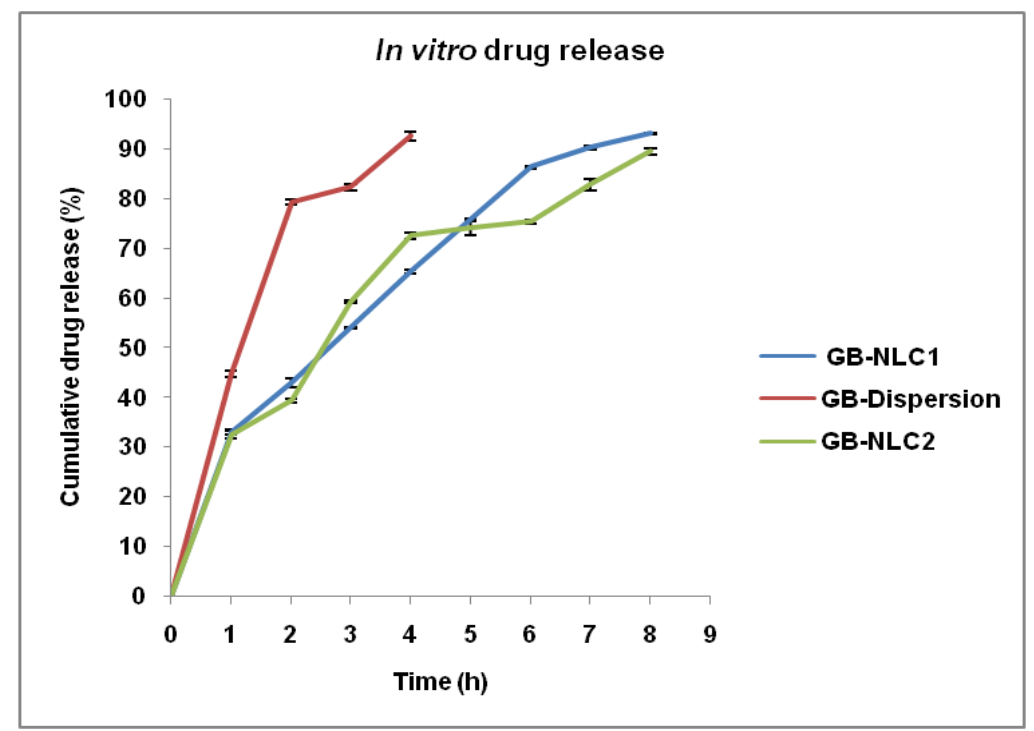

Fig. 12: In vitro drug release profile (mean $\pm S D, n=3$ )

\section{CONCLUSION}

Hot homogenization technique with ultrasonication and melt dispersion technique were successfully utilized for the preparation of nanostructured lipid carriers. The influence of various parameters like sonication time, homogenization speed, stirring rate and temperature for hot homogenization technique and stirring speed, stirring time, and dilution volume for melt dispersion technique on particle size and polydispersity index were methodically studied. Optimized parameters generated by the design were employed to prepare glibenclamide NLC. The resultant GB-NLC was gauged in terms of particle size, electrostatic stability, morphology, the crystallinity of the lipid matrix, and drug release profile. The affirmative outcome of the study indicated the appropriateness of the above design.

The custom design approach was found to be an effective method for obtaining optimized processing parameters for the preparation of NLCs. Dependent variables showing the significant influence on particle size and poly dispersibility index were identified and controlled to obtain optimized formulation. Also the hot homogenization and melt dispersion scheme illustrated to produce NLCs with good drug loading, controlled release profile, and adequate stability.

\section{ACKNOWLEDGEMENT}

The authors are thankful to Mankind Pharma, India, for providing the gift sample of Glibenclamide. Venus Ethoxyethers Pvt. Ltd India is gratefully recognized for providing the gift samples of excipients employed in the studies and Krupanidhi College of Pharmacy for providing the facilities and infrastructure to carry out this work.

\section{FUNDING}

Nil

\section{AUTHORS CONTRIBUTIONS}

All the author have contributed equally.

\section{CONFLICT OF INTERESTS}

The authors declare no conflict of interest.

\section{REFERENCES}

1. Beloqui A, Solinis MA, Rodriguez AG, Almeida AJ, Preat V. Nanostructured lipid carriers: promising drug delivery systems for future clinics. Nanomedicine 2016;12:143-61.
2. Shi F, Wei Z, Zhao Y, Xu X. Nanostructured lipid carriers loaded with baicalin: an efficient carrier for enhanced antidiabetic effects. Pharmacogn Mag.2016;12:198-202.

3. Pardeike J, Hommoss A, Muller RH. Lipid nanoparticles (SLN, NLC) in cosmetic and pharmaceutical dermal products. Int J Pharm 2009;366:170-84.

4. Negi LM, Jaggi M, Talegaonkara S. Development of protocol for screening the formulation components and the assessment of common quality problems of nano-structured lipid carriers. Int J Pharm 2014;461:403-10.

5. Li Q, Cai T, Huang Y, Xia X, Cole SPC, Cai Y. A review of the structure, preparation and application of NLCs, PNPs, and PLNs. Nanomater (Basel, Switzerland); 2017. Available from: http://www.ncbi.nlm.nih.gov/pubmed/28554993. [Last accessed on 31 May 2018]

6. Ganesan P, Narayanasamy D. Lipid nanoparticles: different preparation techniques, characterization, hurdles and strategies for the production of solid lipid nanoparticles and nanostructured lipid carriers for oral drug delivery. Sustain Chem Pharm 2017;6:37-56.

7. Bhagurkar AM, Repka MA, Murthy SN. A novel approach for the development of a nanostructured lipid carrier formulation by hot-melt extrusion technology. J Pharm Sci 2017;106:1085-91.

8. Gardouh AR, Faheim SH, Nouh AT, Ghorab MM. Influence of formulation factors on the size of nanostructured lipid carriers and nanoemulsions prepared by high shear homogenization. Int J Pharm Pharm Sci 2018;10:61-75.

9. Pimentel Moral S, Teixeira MC, Fernandes AR, Borras Linares I, Arraez Roman D, Martinez Ferez A, et al. Polyphenols-enriched hibiscus sabdariffa extract-loaded nanostructured lipid carriers (NLC): optimization by multi-response surface methodology. J Drug Delivery Sci Technol 2019;49:660-7.

10. Maroju RK, Barash S, Brisbane CE. Evaluation of a biologic formulation using customized design of experiment and novel multidimensional robustness diagrams. J Pharm Sci 2017;2:1-10.

11. Gupta S, Kesarla R, Chotai N, Misra A, Omri A. Systematic approach for the formulation and optimization of solid lipid nanoparticles of efavirenz by high pressure homogenization using design of experiments for brain targeting and enhanced bioavailability. Biomed Res Int 2017;3:1-18.

12. Bhaskar K, Anbu J, Ravichandiran V, Venkateswarlu V, Rao YM. Lipid nanoparticles for transdermal delivery of flurbiprofen: formulation, in vitro, ex vivo and in vivo studies. Lipids Health Dis 2009;8:1-15.

13. Uprit S, Kumar Sahu R, Roy A, Pare A. Preparation and characterization of minoxidil loaded nanostructured lipid 
carrier gel for effective treatment of alopecia. Saudi Pharm 2013;21:379-85.

14. Agrawal M, Saraf S, Saraf S, Dubey SK, Puri A, Patel RJ, et al. Recent strategies and advances in the fabrication of nano lipid carriers and their application towards brain targeting. J Controlled Release 2020;321:372-15.

15. Battaglia L, Gallarate M, Panciani PP, Sapino S, Ugazio E, Sapino $\mathrm{S}$, et al. Techniques for the preparation of solid lipid nano and microparticles focus on cerebrospinal fluid: From basic to clinical research view project role of nitric oxide in neurosurgical diseases view project techniques for the preparation of solid lipid nano and microparticles; 2014. Available from: http://dx.doi.org/10.5772/58405. [Last accessed on 09 Oct 2018].

16. Saroja SP, Preethi Sudheer. Formulation and evaluation of aceclofenac mucoadhesive microspheres for oral controlled drug delivery. Asian J Pharm Clin Res 2019;12:184-90.

17. Poonia N, Kaur Narang J, Lather V, Beg S, Sharma T, Singh B, et al. Resveratrol loaded functionalized nanostructured lipid carriers for breast cancer targeting: Systematic development, characterization and pharmacokinetic evaluation. Colloids Surfaces B 2019;181:756-66.

18. Du W, Li H, Tian B, Sai S, Gao Y, Lan T, et al. Biointerfaces development of nose to-brain delivery of ketoconazole by nanostructured lipid carriers against cryptococcal meningoencephalitis in mice. Colloids Surfaces B 2019;183:110446.

19. Aliasgharlou L, Ghanbarzadeh S, Azimi H, Zarrintan MH, Hamishehkar H. Nanostructured lipid carrier for topical application of n-acetyl glucosamine. Adv Pharm Bull 2016;6:581-7.

20. Saedi A, Rostamizadeh K, Parsa M, Dalali N, Ahmadi N. Preparation and characterization of nanostructured lipid carriers as drug delivery system: Influence of liquid lipid types on loading and cytotoxicity. Chem Phys Lipids 2018;216:65-72.

21. Tofani RP, Sumirtapura YC, Darijanto ST. Formulation, characterisation and in vitro skin diffusion of nanostructured lipid carriers for deoxyarbutin compared to a nanoemulsion and conventional cream. Sci Pharm 2016;84:634-45.
22. Abousamra MM, Mohsen AM. Solid lipid nanoparticles and nanostructured lipid carriers of tolnaftate: design, optimization and in vitro evaluation. Int J Pharm Pharm Sci 2016;8:380-5.

23. Reza MI, Goel D, Gupta RK, Warsi MH. Formulation of ketoconazole loaded nano dispersive gel using swollen micelles technique and its in vitro characterization. Int J Pharm Pharm Sci. 2018;10:162-6.

24. Das S, Ng WK, Tan RBH. Are nanostructured lipid carriers (NLCs) better than solid lipid nanoparticles (SLNs): development, characterizations and comparative evaluations of clotrimazoleloaded SLNs and NLCs. Eur J Pharm Sci 2012;47:139-51.

25. Shimojo AM, Fernandes AR V, Ferreira NRE. Evaluation of the influence of process parameters on the properties of resveratrol-loaded NLC using $2^{2}$ full factorial design. Antioxidants (Basel) 2019;8:272-82.

26. Amdoun R, Khelifi L, Khelifi Slaoui M, Amroune S, Asch M, Assaf-ducrocq $\mathrm{C}$, et al. The desirability optimization methodology; a tool to predict two antagonist responses in biotechnological systems: case of biomass growth and hyoscyamine content in elicited datura starmonium hairy roots. Iran J Biotechnol 2018;16:11-9.

27. Reul R, Fricker G, Curi A. Formulation optimization of itraconazole loaded PEGylated liposomes for parenteral administration by using design of experiments. Int J Pharm 2013;448:189-97.

28. Rajinikanth PS, Chellian J. Development and evaluation of nanostructured lipid carrier-based hydrogel for topical delivery of 5-fluorouracil. Int J Nanomed 2016;11:5067-77.

29. Kiss EL, Berko S, Gacsi A, Kovacs A, Katona G, Soo SJ, et al. Design and optimization of nanostructured lipid carrier containing dexamethasone for ophthalmic use. Pharmaceutics 2019;11:1-10.

30. Das S, Ng WK, Tan RBH. Are nanostructured lipid carriers (NLCs) better than solid lipid nanoparticles (SLNs): development, characterizations and comparative evaluations of clotrimazole-loaded SLNs and NLCs. Eur J Pharm Sci 2012;47:139-51.

31. Jia LJ, Zhang DR, Li ZY, Feng FF, Wang YC, Dai WT, et al. Preparation and characterization of silybin-loaded nanostructured lipid carriers. Drug Delivery 2010;17:11-8. 